\title{
ChemComm
}

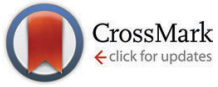

Cite this: Chem. Commun., 2016, 52, 3701

Received 23rd December 2015, Accepted 1st February 2016

DOI: $10.1039 / c 5 c c 10516 a$

www.rsc.org/chemcomm

\section{Nanoparticle-enhanced fluorescence emission for non-separation assays of carbohydrates using a boronic acid-alizarin complex $\dagger$}

\author{
Qianjin Li, Tripta Kamra and Lei Ye*
}

\begin{abstract}
Addition of crosslinked polymer nanoparticles into a solution of a 3-nitrophenylboronic acid-alizarin complex leads to significant enhancement of fluorescence emission. Using the nanoparticleenhanced boronic acid-alizarin system has improved greatly the sensitivity and extended the dynamic range of separation-free fluorescence assays for carbohydrates.
\end{abstract}

Fluorescence measurement has very high sensitivity and is a powerful analytical method for detecting and quantifying important bioactive molecules in the fields of biology, environmental sciences and food industry. ${ }^{1}$ For fluorescent molecules, direct analytical quantification can be achieved using a simple fluorescence spectrophotometer. For non-fluorescent molecules, labelling with a fluorescent probe often needs to be performed before the target analytes can be quantified. The fluorescence labelling introduces additional derivation steps and is cumbersome for routine chemical analysis. Therefore, the use of fluorogenic or fluorescence quenching systems to measure non-fluorescent molecules has attracted great research interest.

Carbohydrates are widespread and many of them play important roles in living organisms. A number of important carbohydrates need to be measured frequently. As an example, glucose needs to be closely monitored for diabetic patients. In the food industry, saccharide measurement is also important for quality control. For different purposes, the sensitivity and dynamic range of carbohydrate analysis can differ significantly. As carbohydrates have no intrinsic fluorescence, it is not possible to directly quantify carbohydrates using fluorescence spectrometry. Compared to carbohydrate analysis through fluorescence labelling, ${ }^{2}$ a more attractive method is to utilize carbohydrate-induced fluorescence quenching, a method that is faster and easier to adopt for routine analysis. ${ }^{3}$ Here, the fluorescence quenching can be realized by the specific interaction/reaction of the carbohydrates with a fluorescent

Division of Pure and Applied Biochemistry, Lund University, Box 124, 22100 Lund, Sweden.E-mail: lei.ye@tbiokem.lth.se

$\dagger$ Electronic supplementary information (ESI) available: Experimental details and additional figures. See DOI: 10.1039/c5cc10516a receptor or a molecular complex. A number of fluorescent receptors/ molecular complexes have been reported in the literature, for example fluorophore-labelled lectins ${ }^{4}$ and boronic acids, ${ }^{5}$ and fluorescent molecular complexes based on boronic acid and alizarin Red S. ${ }^{6}$ Although several boronic acid-based fluorescent receptors have been described in the literature for carbohydrate sensing, ${ }^{7,8}$ the synthesis of the specialized receptors often involves multi-step reactions and can be very costly. In this regard, the fluorescence assay based on phenylboronic acid (PBA)-alizarin Red S (ARS) appears to be simpler and more flexible for carbohydrate measurements. ${ }^{6}$ Since its first report by Wang and co-workers, ${ }^{6}$ the PBA-ARS system has been adopted by many laboratories to develop fluorescence assays for carbohydrates, anions and metal ions. ${ }^{8}$ At the same time, different approaches have been used to improve the analytical performance of the PBA-ARS system for fluorescent carbohydrate assays. Li and co-workers reported that cationic surfactant-assembled vesicles can increase significantly the fluorescence intensity of the PBA-ARS complex. ${ }^{9}$ Using specially designed micelles and vesicles, the intensity of fluorescence emission from the PBA-ARS complex has been increased by $10-13$ folds.$^{10}$ Because of the significant enhancement of fluorescence emission from the PBA-ARS complex, the non-separation carbohydrate assays have been improved to become more accurate and sensitive. Despite the reported progress with the specialized micelles and vesicles, the level of fluorescence enhancement is still limited, and the fluorescenceenhancing micelles and vesicles can become unstable under non-optimal analytical conditions (e.g. organic co-solvent, elevated temperature, etc.).

In this work we study the use of crosslinked polymer nanoparticles as a new type of fluorescence enhancer for non-separation carbohydrate assays based on the general PBA-alizarin system. The surface polarities of the polymer nanoparticles were adjusted so that the nanoparticles could have different affinities for the PBA-ARS complex and lead to different effects of fluorescence enhancement. Compared to previous surfactant-based micelles and vesicles, the polymer nanoparticles developed in this work are much more stable and can be used under more demanding analytical conditions. 
Table 1 Synthesis of crosslinked polymer nanoparticles

\begin{tabular}{llll}
\hline Polymer & Monomer & Crosslinker & Solvent \\
\hline polyMBAA & - & MBAA $(50 \mathrm{mg})$ & Ethanol $(5 \mathrm{~mL})$ \\
Poly(Vpy-co-EGDMA) & Vpy $(14 \mu \mathrm{L})$ & EGDMA $(380 \mu \mathrm{L})$ & Acetonitrile $(9 \mathrm{~mL})$ \\
PolyEGDMA & - & EGDMA $(380 \mu \mathrm{L})$ & Acetonitrile $(9 \mathrm{~mL})$
\end{tabular}

The optimized nanoparticles also provide significantly higher fluorescence enhancement and lead to greatly improved sensitivity and dynamic range for the fluorescent carbohydrate assay.

Three types of polymer nanoparticles were synthesized by one-pot precipitation polymerization (for synthetic details, see the ESI $\dagger$ ). ${ }^{11}$ Different monomer mixtures were used in the free radical polymerization (Table 1 ). The polymer particles were designed to display an increasing hydrophilicity in the order of polyEGDMA $<$ poly(Vpy-co-EGDMA) $<$ polyMBAA. As shown in Fig. 1, all the polymers were obtained in the form of discrete particles with an average particle size smaller than $1 \mu \mathrm{m}$, which was necessary for keeping the particle suspension free from significant sedimentation during a single fluorescence measurement. After being dispersed in aqueous buffer, the polymer particles displayed different emulsion stabilities: while polyMBAA particles remain well dispersed for a prolonged period, the most hydrophobic polyEGDMA formed aggregates and precipitated after standing for $1 \mathrm{~min}$. For the particles with the intermediate polarity, poly(Vpy-co-EGDMA), the particle suspension remained stable for at least 5 min without noticeable sedimentation (see Fig. S1, ESI $\dagger$ ).

When the synthesized polymer nanoparticles were added to an aqueous solution of 3-nitrophenylboronic acid (NPBA) and alizarin, a significant increase ( $\sim 50$ folds) of fluorescence emission was observed for polyEGDMA and poly(Vpy-co-EGDMA) particles (Fig. 2a). The enhancement of fluorescence by the most hydrophilic polyMBAA was moderate. The enhancement of fluorescence is attributed to the binding of the NPBA-alizarin complex to polyEGDMA and poly(Vpy-co-EGDMA) particles, presumably due to hydrophobic interactions. Indeed, when the same polymer
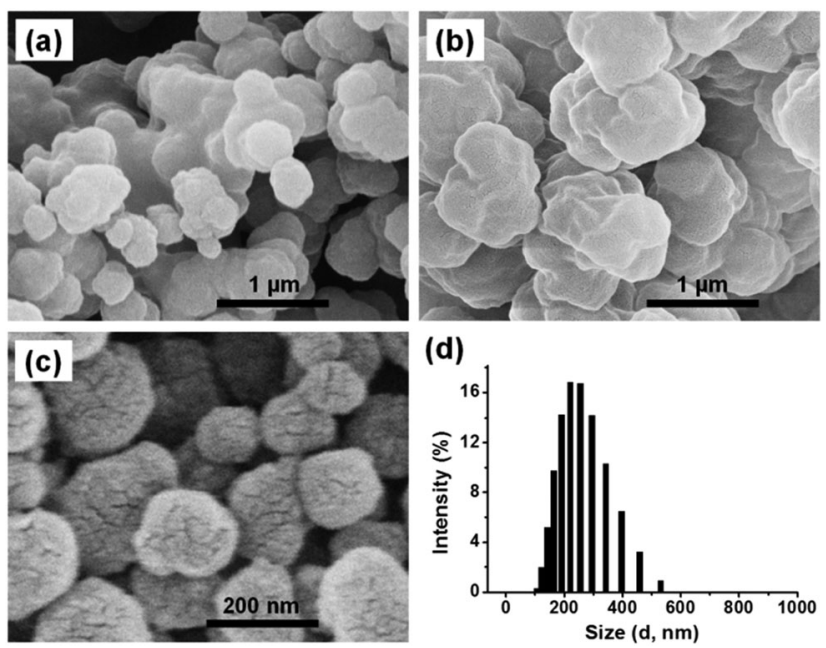

(d)

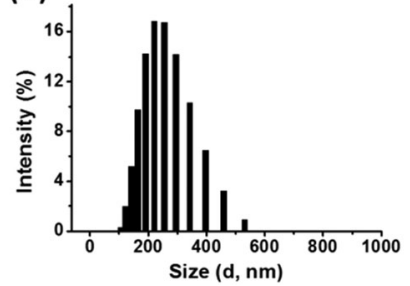

Fig. 1 SEM images of polyMBAA (a), polyEGDMA (b) and poly(Vpy-coEGDMA) (c). (d) Hydrodynamic size of poly(Vpy-co-EGDMA) measured by DLS. (a)

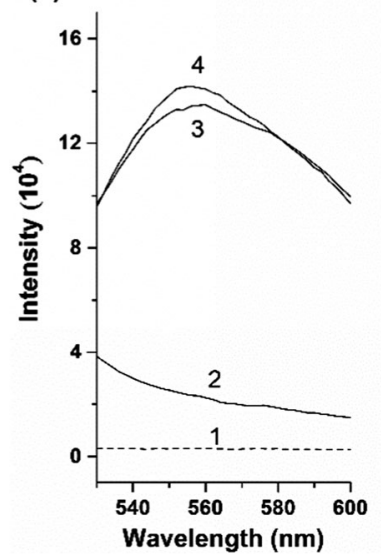

(b)

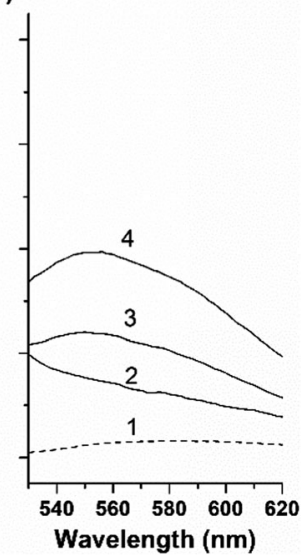

Fig. 2 Fluorescence emission of (a) NPBA-alizarin and (b) NPBA-ARS in phosphate buffer (50 mM, pH 8.0) measured using an excitation wavelength of $467 \mathrm{~nm}$. The spectra were collected from samples containing (1) no particles, (2) polyMBAA, (3) poly(Vpy-co-EGDMA) and (4) polyEGDMA particles. The concentrations of the substances used: NPBA $50 \mu \mathrm{M}$, alizarin $10 \mu \mathrm{M}$, ARS $10 \mu \mathrm{M}$, polymer particles $1 \mathrm{mg} \mathrm{mL}^{-1}$.

particles were mixed with NPBA and the more hydrophilic ARS, the effect of fluorescence enhancement caused by polyEGDMA and poly(Vpy-co-EGDMA) became much smaller (Fig. 2b). We should mention that the fluorescence enhancement is not caused by the basic pyridine moiety alone, because addition of pyridine into NPBA-alizarin solution did not affect the fluorescence emission of the NPBA-alizarin solution (see Fig. S2, ESI $\dagger$ ). Considering the colloidal stability and the effect of fluorescence enhancement, we selected poly(Vpy-co-EGDMA) particles and the NPBA-alizarin system in the remaining studies.

To investigate the relationship between molecular adsorption and fluorescence enhancement, we studied the adsorption of NPBA, alizarin and the NPBA-alizarin complex on poly(Vpy-co-EGDMA) nanoparticles. As shown in Fig. S3 (ESI $\dagger)$, while alizarin $(10 \mu \mathrm{M})$ adsorbed almost quantitatively on the nanoparticles $\left(1 \mathrm{mg} \mathrm{mL}^{-1}\right)$, the adsorption of NPBA $(50 \mu \mathrm{M})$ on the same amount of nanoparticles was only about $18 \%$. However, in the presence of alizarin $(10 \mu \mathrm{M})$, the adsorption of NPBA $(50 \mu \mathrm{M})$ on the nanoparticles increased dramatically, due to that a significant fraction of NPBA formed boronate ester bonds with alizarin, and the NPBA-alizarin complex became adsorbed on the polymer nanoparticles. We should note that no fluorescence enhancement was observed when either NPBA or alizarin solution alone was mixed with the polymer nanoparticles. The result of this semi-quantitative analysis suggests that the adsorption of the NPBA-alizarin complex is the reason for the nanoparticle-enhanced fluorescence emission.

To find out if hydrophobic binding was the underling mechanism for the particle-enhanced fluorescence emission, 


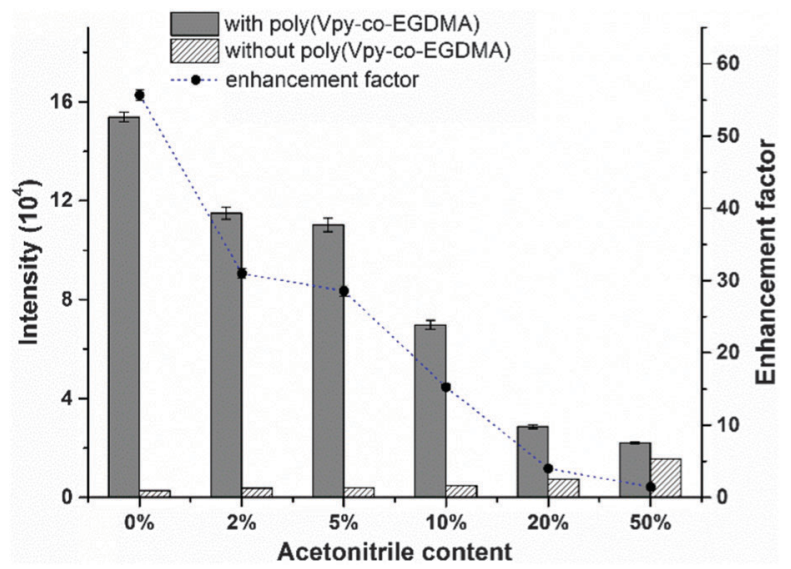

Fig. 3 Effect of organic co-solvent on the fluorescence intensity of NPBA-alizarin solution in phosphate buffer $(50 \mathrm{mM}, \mathrm{pH}$ 7.4). The concentrations of the substances used: alizarin $10 \mu \mathrm{M}$, NPBA $50 \mu \mathrm{M}$, polymer particles $1 \mathrm{mg} \mathrm{mL}^{-1}$. Fluorescence intensity was measured at $558 \mathrm{~nm}$ using an excitation wavelength at $467 \mathrm{~nm}$.

we investigated the effect of adding organic co-solvent into the measurement solutions. As shown in Fig. 3, when an increasing amount of acetonitrile was introduced, the fluorescence-enhancing effect of poly(Vpy-co-EGDMA) decreased. This reduction of fluorescence emission can be explained as a result of decreased NPBAalizarin binding caused by the added acetonitrile. In fact, acetonitrile itself could slightly increase the fluorescence intensity in the particle-free system, which is in agreement with the general effect of organic solvent on fluorescence emission from organic dyes.

Besides the evidence obtained from studying the effect of organic co-solvent, we also tested the same NPBA-alizarin system with more hydrophilic nanoparticles to observe the change in the fluorescence emission (Fig. S4, ESI†). When hydrophilic amino-functionalized silica nanoparticles $\left(\mathrm{Si}-\mathrm{NH}_{2}\right)$ were added, the fluorescence intensity of NPBA-alizarin solution did not change. On the other hand, addition of the more hydrophobic poly(MAA-co-TRIM) nanoparticles increased the fluorescence emission, although the observed fluorescence enhancement is about half of that obtained with the poly(Vpy-co-EGDMA) particles. More importantly, the level of fluorescence enhancement caused by the different nanoparticles follows the order of their capabilities of adsorbing the fluorescent complex (Fig. S3, ESI $\dagger$ ). The largest disparity between molecular adsorption and fluorescence emission is observed for the poly(MAA-co-TRIM) particles. In this case it is likely that the acidic groups on the particle surface provide a local environment that reduced the fluorescence emission. This argument is supported by the fact that fluorescence emission from the nanoparticle-NPBA-alizarin system is $\mathrm{pH}$-sensitive. The optimal $\mathrm{pH}$ value for strong fluorescence emission is in the neutral to weak alkaline range (pH 7.0-8.5) (Fig. S5, ESI $\dagger$ ).

As the optimal poly(Vpy-co-EGDMA) nanoparticles can be used to greatly enhance the fluorescence emission from a NPBA-alizarin solution, it should be possible to extend the dynamic range of carbohydrate assays using the NPBA-alizarin complex because fluorescence variation caused by more concentrated carbohydrate solution can be monitored. To confirm this possibility, we used the

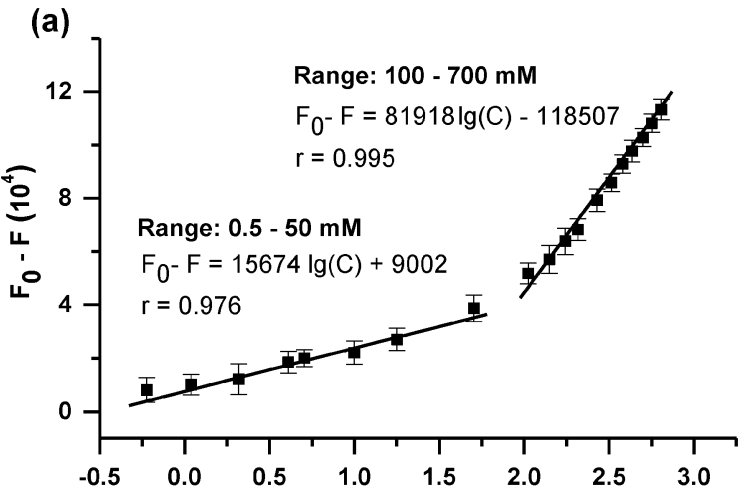

(b)

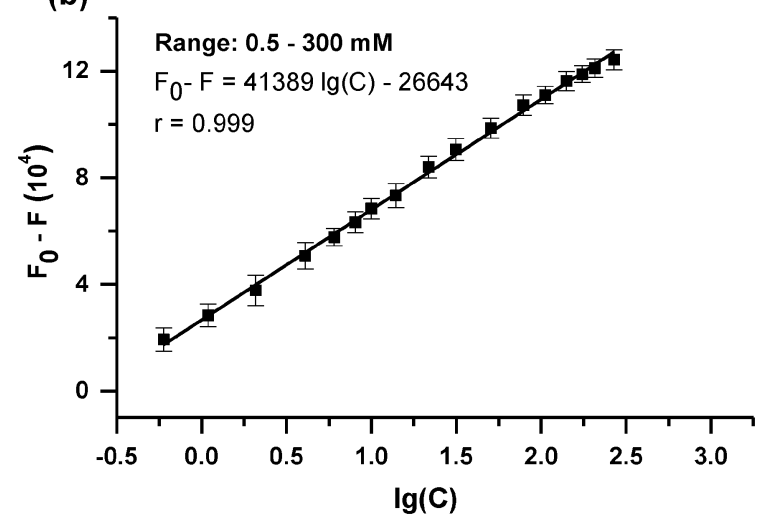

Fig. 4 Response curves of carbohydrate assays obtained by plotting $F_{0}-F$ vs. $\lg (C)$ for glucose (a) and fructose (b). $F_{0}$ and $F$ are the fluorescence intensities of the poly(Vpy-Co-EGDMA)-NPBA-alizarin system measured in the absence and presence of the carbohydrates, respectively. C, concentration of the carbohydrates.

optimal nanoparticle-NPBA-alizarin system as a fluorescent chemical sensor to quantify two simple carbohydrates, glucose and fructose. The competitive assay is based on the following principle: the carbohydrates added into the nanoparticle-NPBA-alizarin system displace alizarin from NPBA, leading to a decreased fluorescence emission. In Fig. 4 we show the dose-response curves obtained from the competitive assays for glucose and fructose.

In Fig. 4a, the fluorescence reduction caused by glucose displays two different linear ranges, which differs from the response of fructose (Fig. 4b). The reason for the two separate linear ranges for glucose assays is not clear, but may be related to the fact that glucose has a weaker boronic acid affinity and can bind to NPBA at two different cis-diol moieties. ${ }^{12}$ We should mention that the two different dynamic ranges for glucose assays are also observed in the particle-free NPBA-alizarin system (Fig. S6a, ESI $\dagger$ ), indicating that the phenomenon is not caused by the polymer nanoparticles.

The sensitivity of the competitive carbohydrate assay can be evaluated using the slope of the response curves in Fig. 4. In the presence of poly(Vpy-co-EGDMA) nanoparticles, the slope for glucose assays in the low and high concentration ranges are about 34 and 50 times higher than that obtained from the particle-free NPBA-alizarin system (Fig. S6a, ESI $\dagger$ ), respectively. For fructose, the slope of the response curve obtained from the 
poly(Vpy-co-EGDMA)-NPBA-alizarin system (Fig. 4b) is about 34 times higher than that from the particle-free NPBA-alizarin system (Fig. S6b, ESI $\dagger$ ). These results demonstrate that addition of poly(Vpy-co-EGDMA) nanoparticles increased significantly the sensitivity of the NPBA-alizarin system for the carbohydrate assay.

Using the poly(Vpy-co-EGDMA)-NPBA-alizarin system for glucose detection, obvious fluorescence response was found in the concentration range of $0.5-700 \mathrm{mM}$, which is 10 times wider than that achieved with the particle-free NPBA-alizarin system (glucose concentration $0.5-80 \mathrm{mM}$, Fig. S4a, ESI $\dagger$ ). For fructose detection, the response range is extended by 30 folds to cover the fructose concentration up to $300 \mathrm{mM}$ (Fig. S4b, ESI $\dagger$ ). In view of the significantly widened detection range, the poly(Vpy-co-EGDMA)-NPBA-alizarin system should be useful not only for monitoring glucose in human body fluid (normal glucose concentration in human blood for two hours after meals $<7.8 \mathrm{mM}$, diabetic patient $>11.1 \mathrm{mM}){ }^{13}$ but also for direct detection of carbohydrates in, e.g. food, beverage, and for monitoring fermentation processes.

In this work we have developed a nanoparticle enhanced fluorescent system for carbohydrate assays using NPBA-alizarin as a fluorescent reporter. The fluorescent system is easy to prepare and operate, and it exhibits several advantages including not requiring any separation step, having high sensitivity and allowing bioactive cis-diols to be directly measured in a broader concentration range. Based on these advantages, the developed nanoparticle enhanced fluorescent system may find interesting applications for measuring carbohydrates directly in different samples, for example in the fields of diagnostics, biomedicine and for monitoring food production processes.

This work was supported by the Swedish Research Council FORMAS (grant no. 212-2013-1350).

\section{Notes and references}

1 J. Yao, M. Yang and Y. X. Duan, Chem. Rev., 2014, 114, 6130; R. K. Henderson, A. Baker, K. R. Murphy, A. Hamblya, R. M. Stuetz and S. J. Khan, Water Res., 2009, 43, 863; S. A. Eremin, Food Technol. Biotechnol., 1998, 36, 235.

2 K. R. Anumula, Anal. Biochem., 2006, 350, 1.

3 N. DiCesare, M. R. Pinto, K. S. Schanze and J. R. Lakowicz, Langmuir, 2002, 18, 7785.

4 I. Hamachi, T. Nagase and S. Shinkai, J. Am. Chem. Soc., 2000, $122,12065$.

5 T. D. James, K. Sandanayake and S. Shinkai, Nature, 1995, 374, 345.

6 G. Springsteen and B. H. Wang, Chem. Commun., 2001, 1608.

7 T. D. James, K. Sandanayake and S. Shinkai, Angew. Chem., Int. Ed., 1996, 35, 1910; G. Springsteen and B. H. Wang, Tetrahedron, 2002, $58,5291$.

8 X. Sun and T. D. James, Chem. Rev., 2015, 115, 8001.

9 Q. Wang, G. Li, W. Mao, H. Qi and G. Li, Sens. Actuators, B, 2006, 119, 695.

10 K. Ngamdee, T. Noipa, S. Martwiset, T. Tuntulani and W. Ngeontae, Sens. Actuators, B, 2011, 160, 129; O. Savsunenko, H. Matondo, S. Franceschi-Messant, E. Perez, A. F. Popov, I. Rico-Lattes, A. Lattes and Y. Karpichev, Langmuir, 2013, 29, 3207.

11 K. Yoshimatsu, K. Reimhult, A. Krozer, K. Mosbach, K. Sode and L. Ye, Anal. Chim. Acta, 2007, 584, 112.

12 V. V. Karnati, X. M. Gao, S. H. Gao, W. Q. Yang, W. J. Ni, S. Sankar and B. H. Wang, Bioorg. Med. Chem. Lett., 2002, 12, 3373.

13 K. Alberti and P. Z. Zimmet, Diabetic Med., 1998, 15, 539; Definition and diagnosis of diabetes mellitus and intermediate hyperglycemia: report of a WHO/IDF consultation. WHO, 2006. 